\title{
Formation of Methane Hydrate in the Presence of Natural and Synthetic Nanoparticles
}

Stephen J. Cox, ${ }^{\dagger \neq}$ Diana J. F. Taylor, ${ }^{\ddagger, I L}$ Tristan G. A. Youngs, ${ }^{\S}$ Alan K. Soper, ${ }^{\S}$ Tim S. Totton, Richard G. Chapman," Mosayyeb Arjmandi," Michael G. Hodges, "Neal T. Skipper,*,+,II and Angelos Michaelides*,+, If

${ }^{\dagger}$ Department of Chemistry, University College London, 20 Gordon Street, London WC1H 0AJ, United Kingdom

¥Thomas Young Centre and London Centre for Nanotechnology, 17-19 Gordon Street, London WC1H 0AH, United Kingdom

IIDepartment of Physics and Astronomy, University College London, Gower Street, London WC1E 6BT, United Kingdom

${ }^{\S}$ ISIS Facility, STFC Rutherford Appleton Laboratory, Harwell Oxford, Didcot OX11 0QX, United Kingdom

"BP Exploration Operating Co. Ltd, Chertsey Road, Sunbury-on-Thames TW16 7LN, United Kingdom

Supporting Information

ABSTRACT: Natural gas hydrates occur widely on the ocean-bed and in permafrost regions, and have potential as an untapped energy resource. Their formation and growth, however, poses major problems for the energy sector due to their tendency to block oil and gas pipelines, whereas their melting is viewed as a potential contributor to climate change. Although recent advances have been made in understanding bulk methane hydrate formation, the effect of impurity particles, which are always present under conditions relevant to industry and the environment, remains an open question. Here we present results from

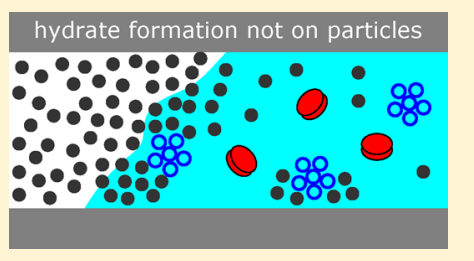
neutron scattering experiments and molecular dynamics simulations that show that the formation of methane hydrate is insensitive to the addition of a wide range of impurity particles. Our analysis shows that this is due to the different chemical natures of methane and water, with methane generally excluded from the volume surrounding the nanoparticles. This has important consequences for our understanding of the mechanism of hydrate nucleation and the design of new inhibitor molecules.

\section{INTRODUCTION}

The clathrate hydrates of natural gases are crystalline compounds in which gas molecules are encaged in a host framework of water molecules. These materials form under high pressure and low temperature, and occur naturally on the ocean bed and in permafrost. ${ }^{1}$ It is estimated that the total amount of hydrated gas on Earth exceeds conventional gas reserves by at least an order of magnitude. ${ }^{2}$ This makes natural gas hydrates not only a potential untapped energy resource but also a historic and contemporary source of greenhouse gases ${ }^{3}$ (methane is 21 times more powerful a greenhouse gas than carbon dioxide). Natural gas hydrates also pose a severe problem in oil and gas pipelines: if the mixed phases of water and natural gas are allowed to cool, hydrates may form and block the line, causing production to stall. ${ }^{1}$ The consequences of this are becoming more severe as extraction from deeper oil and gas reserves becomes more commonplace. Chemicals for inhibiting hydrate nucleation and growth exist. However, they have generally been found on a trial-and-error basis, and it remains unclear how they work at the molecular scale. Nonetheless, improved understanding of the molecular level processes that govern gas hydrate formation is essential for the systematic design of future inhibitor technologies. ${ }^{4,5}$ More broadly, hydrate formation is an archetypal example of a nucleation process involving two chemical components; better understanding of this process could therefore be relevant to improving insight into nucleation in general.

The industrial and environmental importance of gas hydrates has motivated many studies into the mechanisms by which they form. ${ }^{6-35}$ In particular, methane hydrate is one of the most commonly studied natural gas hydrates owing both to its natural abundance and importance in "lean" gas lines. Moreover, the fact that methane is one of the simplest hydrophobes makes it an appealing model system to understand gas hydrate formation more generally. Historically, two main molecular mechanisms for hydrate nucleation have been proposed: (i) the "labile cluster hypothesis" (LCH), that describes the nucleation process as the formation of isolated hydrate cages, which then agglomerate to form a critical hydrate nucleus; $; 3,37$ and (ii) the "local structure hypothesis" (LSH), in which the guest molecules first arrange themselves in a structure similar to the hydrate phase, followed by a rearrangement of water around the locally ordered guest molecules. ${ }^{38}$ Simulation studies of homogeneous methane hydrate nucleation have suggested a mechanism somewhere between the LCH and LSH. ${ }^{, 10}$ Similarly, neutron scattering and ${ }^{13} \mathrm{C}$ magic angle spinning NMR experiments ${ }^{7,39}$ have found that long-lived hydration shells around dissolved methane

Received: November 14, 2017

Published: February 5, 2018 
molecules are dynamical rather than rigid clathrate-like structures, and that water structure only changes substantially once the hydrate has formed. Although open questions remain, these previous experimental and simulation studies exemplify the recent progress made in our understanding of hydrate formation in "pure" (i.e., gas + water) systems. For a more detailed overview of current opinion on the underlying mechanisms of hydrate formation, we refer the reader to the recent review article by Warrier et al. ${ }^{5}$

Here we focus exclusively on methane hydrate, specifically with the aim to understand the effect of dissolved solid impurity particles on its formation mechanism. This is motivated, in part, by the previous work of Knott et al., who investigated the homogeneous nucleation of methane hydrate with computer simulation. ${ }^{33}$ Using the "seeding technique" to determine the relevant parameters in the classical nucleation theory (CNT) rate expression, Knott et al. computed a nucleation rate on the order of $10^{-111}$ nuclei $\mathrm{cm}^{-3} \mathrm{~s}^{-1}$. As the conditions under consideration were $273 \mathrm{~K}$ and $900 \mathrm{~atm}$, and at reasonable supersaturations of dissolved methane, it was concluded that methane hydrate must form, not homogeneously, but via a heterogeneous nucleation mechanism. Aside from seafloor sediment, permafrost, pipelines, and high-pressure lab equipment, it was speculated that mineral surfaces could act as catalysts for methane hydrate formation. A systematic study of the effect of solid particles on the formation of methane hydrate will help shed light onto the mechanism by which this important process occurs.

In this article, we use neutron scattering in conjunction with hydrogen-deuterium isotopic labeling experiments to investigate methane hydrate formation in the presence of a wide variety of solid nanoparticles. Much to our surprise, we find that the kinetics of formation of methane hydrate is insensitive to the addition of these impurities. We also present results of molecular dynamics (MD) simulations to probe the molecular mechanisms by which methane hydrate forms in the presence of solid surfaces, and find that nucleation happens away from the solid/liquid interface in all cases studied. Our results suggest that, with regard to designing improved inhibitors, it may be advantageous to focus on understanding the mechanism of methane hydrate formation either in the bulk, or at the gas/ liquid interface.

The remainder of the article is organized as follows. In section 2, we describe the experimental and simulation methods. We then present results first from the neutron scattering experiments, and then from the MD simulations in section 3. These results are then discussed in section 3.3, and conclusions are presented in section 4 .

\section{METHODS}

2.1. Time-Resolved Neutron Scattering Experiments. Methane dissolution and hydrate formation have been studied simultaneously by exploiting time-resolved neutron diffraction in conjunction with $\mathrm{H} / \mathrm{D}$ isotopic labeling. Experiments were conducted on the NIMROD $^{40}$ and SANDALS ${ }^{41}$ time-of-flight neutron diffractometers at the ISIS pulsed neutron source, STFC Rutherford Appleton Laboratory (Didcot, UK). These instruments are optimized for studies of liquids and amorphous materials containing a high proportion of hydrogen $\left({ }^{1} \mathrm{H}\right)$, and they provide continuous access to a momentum transfer range $0.02<Q<50 \AA^{-1}$.

The hydrate samples were prepared in situ on the beamline in a cylindrical geometry null scattering titanium/zirconium alloy pressure cell, of the type originally developed by Buchanan et al. ${ }^{6}$ This cell has height $40 \mathrm{~mm}$, inner diameter $15 \mathrm{~mm}$ and wall thickness $3 \mathrm{~mm}$. The bottom of this cell has a dead-volume that contains a $10 \mathrm{~mm}$ steel ball bearing, and the entire cell system can be inverted with a frequency of ca. $0.5 \mathrm{~Hz}$ in the neutron beam to allow mixing of the sample and the pressurizing methane gas (see Figure S1). Temperature was controlled to within $\pm 0.05{ }^{\circ} \mathrm{C}$ via a circulating water-glycol heat bath. For each experiment the sealed/evacuated sample cell was first loaded with 8.3 $\mathrm{cm}^{3}$ of liquid $\left(\mathrm{D}_{2} \mathrm{O}\right.$ or $\mathrm{D}_{2} \mathrm{O}+$ clay/silica $)$ via a bleed-in pipe at the cell base. Pressurized methane $\left(\mathrm{CH}_{4}\right)$ was then introduced over the liquid using a pressurized gas hand pump. Standard working conditions were 180 bar methane and $278 \mathrm{~K}$. At this working pressure, sI methane hydrate is stable below $293 \mathrm{~K}^{6}$ Methane pressure was maintained during the experiment by top-up from the hand-pump.

To ensure thorough and reproducible mixing of the methane gas and solution, we employed two agitation regimes, referred to as "standard" and "short". Both of these agitation regimes are shown schematically in Figure S1. The first stage in both regimes was "preproduction", which consisted of 15 min data collection, followed by 15 min shaking, then another $15 \mathrm{~min}$ data collection, before cooling over a $30 \mathrm{~min}$ interval from 298 to $278 \mathrm{~K}$. In the standard regime, we then performed the following: 'stage 0', $15 \mathrm{~min}$ data collection; 'stage 1', 1 shake ( $2 \mathrm{~s})$ then $15 \mathrm{~min}$ data collection; 'stage 2', 10 shakes (20 s) then $15 \mathrm{~min}$ data collection; 'stage 3', 100 shakes (200 s) then $15 \mathrm{~min}$ data collection; and 'stage 4', 450 shakes $(900 \mathrm{~s})$ then $15 \mathrm{~min}$ data collection. We specify our time origin $(t=0)$ immediately after stage 0 . We define the "standard agitation time", $t_{\mathrm{a}, \mathrm{st}} \approx 80 \mathrm{~min}$, as the time immediately after stage 4 . Data were then collected in $15 \mathrm{~min}$ intervals. All results in the main paper are obtained with this standard agitation regime. The short regime is used for further control experiments and is presented in the Supporting Information (S.I.), where further experimental details can also be found.

The samples studied are summarized in Table 1 . The clays we used are in the 2:1 family, for which the end members are talc and

Table 1. Clay and Silica Nanoparticle Samples Studied by Neutron Scattering (wt \% shown in parentheses) ${ }^{a}$

\begin{tabular}{|c|c|c|c|c|c|}
\hline Sample & Morphology & C.I. & $\sigma_{\text {surf }}$ & $\zeta$ & $d$ \\
\hline $\begin{array}{l}\text { Vermiculite Eucatex } \\
\text { clay }(0.5)\end{array}$ & Plates $(0.92,-)$ & $\mathrm{C}_{3} \mathrm{H}_{7} \mathrm{NH}_{3}^{+}$ & -0.21 & - & - \\
\hline $\begin{array}{l}\text { Laponite B clay } \\
(0.5)\end{array}$ & Discs $(0.92,25)$ & $\mathrm{Na}^{+}$ & -0.12 & - & 40 \\
\hline $\begin{array}{l}\text { Laponite B clay } \\
(2.0)\end{array}$ & $\operatorname{Discs}(0.92,25)$ & $\mathrm{Na}^{+}$ & -0.12 & - & 25 \\
\hline $\begin{array}{l}\text { Laponite } \mathrm{RD} \text { clay } \\
\quad(0.5)\end{array}$ & Discs $(0.92,25)$ & $\mathrm{Na}^{+}$ & -0.12 & -42.3 & 40 \\
\hline $\begin{array}{l}\text { Laponite RD clay } \\
\quad(2.0)\end{array}$ & Discs $(0.92,25)$ & $\mathrm{Na}^{+}$ & -0.12 & -42.3 & 25 \\
\hline $\begin{array}{l}\text { Smectite SWy-2 } \\
\text { clay }(0.5)\end{array}$ & Plates $(0.92,-)$ & $\mathrm{Na}^{+}$ & -0.10 & -37.0 & - \\
\hline $\begin{array}{l}\text { Silica nanospheres } \\
(0.5)\end{array}$ & Spheres $(-, 20)$ & $\mathrm{H}_{3} \mathrm{O}^{+}$ & - & -45.3 & 80 \\
\hline $\begin{array}{l}\text { Silica nanospheres } \\
\quad(0.5)\end{array}$ & Spheres $(-, 80)$ & $\mathrm{H}_{3} \mathrm{O}^{+}$ & - & -33.7 & 31 \\
\hline $\begin{array}{l}\text { Silica nanospheres } \\
\quad(0.1)\end{array}$ & Spheres $(-, 80)$ & $\mathrm{H}_{3} \mathrm{O}^{+}$ & - & -33.7 & 5 \\
\hline $\begin{array}{l}\text { Silica nanospheres } \\
(0.02)\end{array}$ & Spheres $(-, 80)$ & $\mathrm{H}_{3} \mathrm{O}^{+}$ & - & -33.7 & 9 \\
\hline
\end{tabular}

${ }^{a}$ All nanoparticles were dissolved in $\mathrm{D}_{2} \mathrm{O}$, and the control had no added nanoparticles. Information on the morphology (thickness, diameter $(\mathrm{nm})$ shown in parentheses), counterion (C.I.), surface charge $\left(\sigma_{\text {surf }}\right.$ in $\left.\mathrm{C} \mathrm{m}^{-2}\right), \zeta$-potential $(\zeta$, in $\mathrm{mV})$ and average particle separation $(d$, in $\mathrm{nm})$ is also given, where available. See the S.I. for further details and results from additional control measurements.

pyrophyllite (uncharged, hydrophobic) and mica (highly charged, hydrophilic). To ensure dispersion of the clays, we typically prepared them in sodium substituted form, with the exception of the high charge vermiculite which was prepared with propylammonium.

All measurements were made in heavy water $\mathrm{D}_{2} \mathrm{O}$ as this provides a strong coherent signal and avoids the high background resulting from 
incoherent scattering from $\mathrm{H}_{2} \mathrm{O}$. Physically, all of the systems are lowviscosity liquids, with the exception of 2 wt \% Laponite B which is a strong gel former, and 2 wt \% Laponite $\mathrm{RD}$ which forms a thixotropic gel over $c a .6 \mathrm{~h}$. In the case 2 wt \% Laponite B we conducted agitation over $c a .4 \mathrm{~h}$ in total, with data collection of approximately an hour after each step.

2.2. Molecular Dynamics Simulations. Methane hydrate formation was investigated with coarse grained and all-atom models at a variety of surfaces. Specifically, with the coarse grained model we studied the (111) surface of a face centered cubic (fcc) crystal that interacted with the water by a Lennard-Jones potential, and a graphene sheet. A variety of interaction strengths between the surface atoms and the water molecules was used, giving rise to monomer adsorption energies to the surface in the range $0.80-20.11 \mathrm{kcal} / \mathrm{mol}$. For each surface hydrophilicity, a $100 \mathrm{~ns}$ isothermal-isobaric simulation was performed at $250 \mathrm{~K}$ and $900 \mathrm{~atm}$, which resulted in a phase separated mixture of methane and water. Five initial configurations were selected from the second half of this trajectory, and the velocities were randomized according to the Maxwell-Boltzmann distribution for a temperature of $250 \mathrm{~K}$, and the target temperature of the thermostat was decreased at a rate of $0.1 \mathrm{~K} / \mathrm{ns}$. The equations of motion were integrated until nucleation was observed, which took on the order of $100 \mathrm{~ns}$. We note that although these conditions result in a high driving force for nucleation, similar protocols have been used previously to successfully investigate heterogeneous ice nucleation. ${ }^{42-46}$ This suggests that if the surfaces were to act as catalysts for methane hydrate formation, then this would be observed with the simulation techniques used in this study. Water was modeled using the $\mathrm{mW}$ model. ${ }^{47}$ Methane-methane and methane-water interactions were described by the potential outlined by Jacobson and Molinero, ${ }^{48}$ but using the reparametrization given by Knott et al., ${ }^{33}$ which improves the solubility of methane at higher pressures. All simulations involving $\mathrm{mW}$ used the LAMMPS simulation package, ${ }^{49}$ and consisted of 6846 water and 1154 methane molecules.

Recent simulation studies have shown that both of these surfaces promote ice nucleation. ${ }^{42-46,50,51}$ As others have suggested that ice may facilitate hydrate formation by directly affecting the structure of the interfacial water, ${ }^{24-26}$ and the fact that the hydrate/liquid and ice/ liquid interfaces have been found to exhibit similar surface free energies, ${ }^{33}$ it is interesting to explore hydrate formation in the presence of these surfaces. In the case of the fcc surface, ice nucleation was enhanced by promoting epitaxial growth of the crystal, and given that hexagonal rings of water are a common motif in gas hydrates, there exists a possibility of a similar mechanism in the current context. On the other hand, the graphene surface promotes ice nucleation by inducing layers in the water density normal to the surface, and provides an alternative mechanism to facilitate methane hydrate formation other than epitaxial growth.

For studies with the all-atom models, we have investigated methane hydrate formation in the presence of the clay mineral, kaolinite. The system, consisting of 2944 water and 512 methane molecules, was prepared by melting a hydrate crystal at $425 \mathrm{~K}$ and 400 bar $(394.8$ atm) for $20 \mathrm{~ns}$, resulting in a phase separated system with the methane at the silicate terminated face, and a planar interface separating the methane and water. Initial configurations were then drawn from this trajectory, with the velocities randomized with a target temperature of $245 \mathrm{~K}$. Dynamics were then propagated using the GROMACS 4.5.5 simulation package ${ }^{52}$ at a target pressure of 500 bar. Further details regarding both the coarse-grained and all-atom simulations are given in the SI.

\section{RESULTS}

3.1. Neutron Scattering: Insensitivity of Methane Hydrate Formation to the Presence of Impurity Particles. We have used in situ time-resolved neutron scattering to measure the uptake of protiated methane $\left(\mathrm{CH}_{4}\right)$ into deuterated water $\left(\mathrm{D}_{2} \mathrm{O}\right)$ solutions, and have then followed the subsequent formation of the crystalline methane hydrate sI structure of composition $\mathrm{CH}_{4}: \mathrm{D}_{2} \mathrm{O} \approx 1.0: 5.75$. In addition to the control $\mathrm{CH}_{4}-\mathrm{D}_{2} \mathrm{O}$ system, we have studied these processes in the presence of a variety of dissolved clay and silica nanoparticles (both natural and synthetic, see Table 1), as examples of impurity particles that one might expect to find under natural and industrial conditions. A broad range of particles was considered with different surface structures and hydrophilicities, with a view to exploring generic factors that are postulated to be relevant in heterogeneous nucleation.

For each run, the function that is extracted from the neutron scattering data is known as the differential scattering cross section (DCS), measured in barn steradian ${ }^{-1}$ atom $^{-1}\left(\mathrm{~b} \mathrm{sr}^{-1}\right.$ atom $^{-1}$ ). Of the isotopes in our systems, hydrogen ${ }^{1} \mathrm{H}$ has by far the largest total neutron scattering cross section (see Table S1). The overall level of the DCS can therefore be used to extract the absolute concentration of methane in the samples, and we estimate the ratio of water to methane molecules to be approximately 20:1. Moreover, hydrogen ${ }^{1} \mathrm{H}$ has the only negative scattering length of the species in our samples. This contrast means that the (110), (200), (210) and (211) sI hydrate Bragg peaks are intense in our diffraction patterns, allowing us to monitor, in real time, the growth of the crystalline phase. This is illustrated in Figure 1.

In Figure 1a, we show the effects of agitation on the sample containing $0.5 \mathrm{wt} \%$ Laponite RD solids. We note that, as expected, the DCS scattering level increases rapidly on agitation as methane is dissolved in the solution, but thereafter rises very slowly over a time scale of hours. Comparing samples in Figure $1 \mathrm{~b}$, we see that the total scattering level of the DCS, which reports directly on the dissolved methane concentration, is generally decreased in the presence of 0.5 wt \% solid additives. This suggests that, for the methane, there is a (partially) excluded volume around the solid particles, and certainly no strong adsorption of methane around the solid surfaces. This conclusion is supported by results obtained with higher wt \% Laponite samples, where hydrate formation is severely inhibited (see Figure S4). It should be noted that the presence of nanobubbles of methane gas can be ruled out, due to the lack of small-angle scattering from our solutions.

Analysis of the Bragg peaks allows us to obtain a direct measure of the amount of hydrate crystal in the sample as function of time, in a way that cannot be obtained by monitoring, for example, the methane pressure. Figure la already suggests that, while some hydrate forms during the initial stages of agitation, the crystal growth to equilibrium after agitation takes several hours. This is in direct contrast to the uptake of methane, which is relatively constant after agitation (Figure $2 \mathrm{~b}$ inset, and Figure S5). In this paper, we are interested primarily in the relative kinetics of methane hydrate formation with and without solid additives. To this end, we have normalized our Bragg peak intensities in two different ways. If we denote the total area of the Bragg peaks at time $t$ as $A_{\text {Bragg }}(t)$, then the first of these approaches normalizes by $A_{\mathrm{Bragg}}\left(t_{\mathrm{a}, \mathrm{st}}\right)$, where $t_{\mathrm{a}, \mathrm{st}} \approx 80 \mathrm{~min}$ is the time at the final agitation step. This is shown in Figure 2a. This normalization accentuates the underlying kinetics of crystal growth. In the second approach, shown in Figure $2(\mathrm{~b}), A_{\mathrm{Bragg}}(t)$ is normalized by $\Delta L_{\mathrm{s}}\left(t_{\mathrm{a}, \mathrm{st}}\right) \equiv L_{\mathrm{s}}\left(t_{\mathrm{a}, \mathrm{st}}\right)-L_{\mathrm{s}}(0)$, the scattering level increase at the final agitation step (see Figure S2). This normalization scheme has the advantage of removing the effect of random variations in the methane uptake, which can be up to $10 \%$ when we compare repeats of the same sample (as we have done with the control system of $\mathrm{CH}_{4}$ in pure $\mathrm{D}_{2} \mathrm{O}$ ). From Figure $2 \mathrm{a}$, we see that there is no significant difference between the 

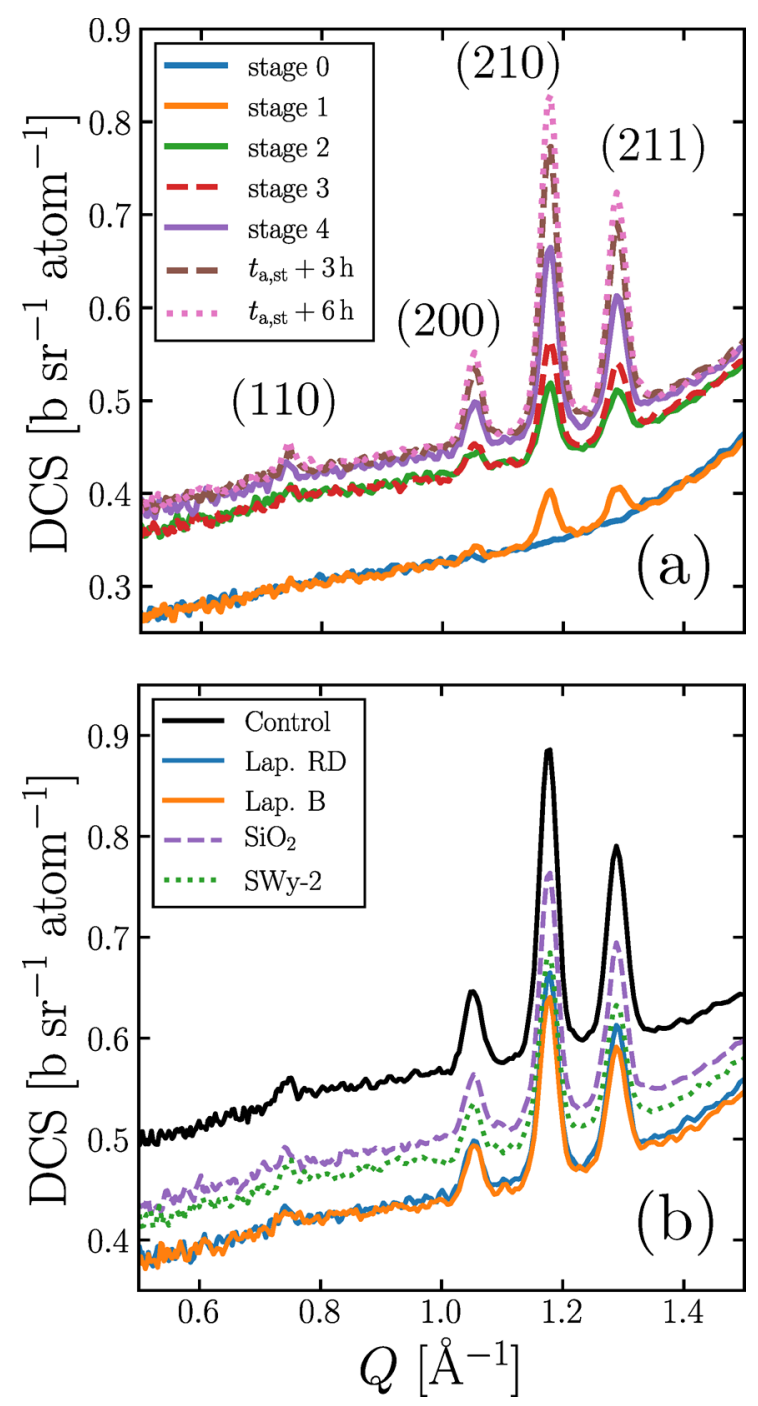

Figure 1. Methane hydrate formation as studied by neutron diffraction from $\mathrm{CH}_{4}+\mathrm{D}_{2} \mathrm{O}$ solutions at 180 bar and $278 \mathrm{~K}$. All results obtained with the "standard" agitation regime. (a) The effects of agitation time for a sample containing 0.5 wt \% Laponite $\mathrm{RD}$ clay, showing the increase of the DCS level (Eq. S2) and evolution of the sI methane hydrate Bragg peaks. It is clear that the overall scattering level increases as methane is dissolved into the $\mathrm{D}_{2} \mathrm{O}$. (b) The effects of different solid additives $(0.5 \mathrm{wt} \%)$ as seen in the DCS at time $t_{\mathrm{a}, \mathrm{st}}$. Note that the scattering level is highest for the control sample of $\mathrm{CH}_{4}+\mathrm{D}_{2} \mathrm{O}$ without added solids. The $\mathrm{SiO}_{2}$ sample consists of $20 \mathrm{~nm}$ spheres.

underlying kinetics of hydrate formation in the control sample or any of the systems with solid additives, while Figure $2 b$ shows that the amount of hydrate formed per unit of dissolved methane is greatest in the control sample without additives. These are key results from our neutron scattering experiments, which are uniquely provided by our combination of isotopic labeling and in situ total scattering. These experimental data therefore suggest that the presence of the particulate matter investigated here has little effect on the formation of methane hydrate. This is a somewhat striking result, especially when one considers that the presence of such particles generally enhances ice formation by orders of magnitude. ${ }^{53,54}$

With our experimental protocol, however, we cannot preclude the formation of methane hydrate at interfaces other than those presented by the mineral particles, such as the surface of the pressure cell or the gas/liquid interface. To test
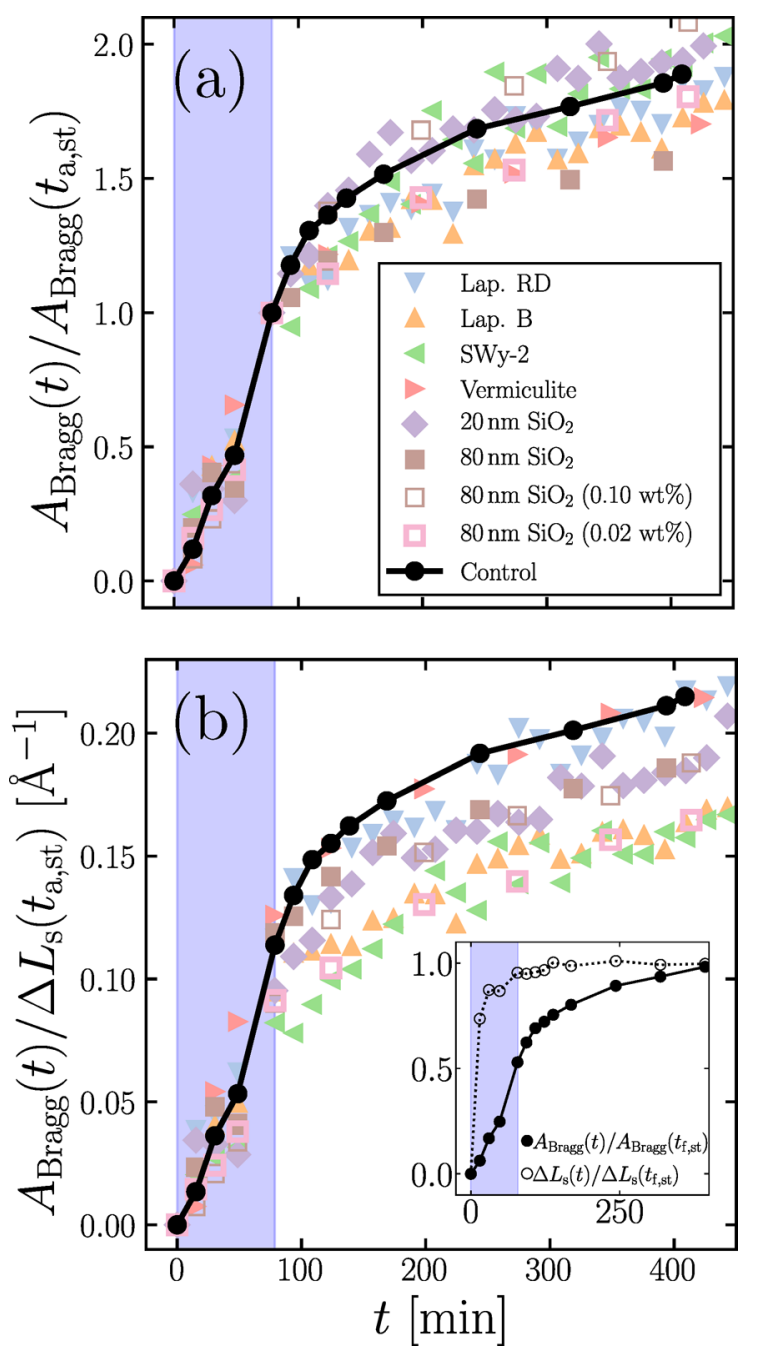

Figure 2. Time evolution of $A_{\mathrm{Bragg}}(t)$, the total area of the (110), (200), (210) and (211) Bragg peaks. The shaded blue regions show the time over which standard agitation was performed. (a) $A_{\mathrm{Bragg}}(t) /$ $A_{\text {Bragg }}\left(t_{\mathrm{a}, \mathrm{st}}\right)$. There is no significant difference between the control and any of the samples in terms of hydrate formation kinetics. (b) $A_{\text {Bragg }}(t) / \Delta L_{\mathrm{s}}\left(t_{\mathrm{a}, \mathrm{st}}\right)$, where $\Delta L_{\mathrm{s}}\left(t_{\mathrm{a}, \mathrm{st}}\right)$ is the scattering level increase at $t_{\mathrm{a}, \mathrm{st}}$ (proportional to the amount of dissolved methane). The quantity of hydrate formed per unit of dissolved methane is either similar to, or less than, the control sample. Inset: Time evolution of $A_{\mathrm{Bragg}}(t) /$ $A_{\text {Bragg }}\left(t_{\mathrm{f}, \mathrm{st}}\right)$ and $\Delta L_{\mathrm{s}}(t) / \Delta L_{\mathrm{s}}\left(t_{\mathrm{f}, \mathrm{st}}\right)$ for the control sample, where $t_{\mathrm{f}, \mathrm{st}}=$ $410 \mathrm{~min}$. This shows that the uptake of methane into solution completes on a much quicker time scale than the evolution of sI hydrate.

whether the surface chemistry of the pressure cylinder intrinsically lends itself to enhancing nucleation (e.g., by promoting particular structures in the liquid), we also conducted further control experiments using the "short" agitation regime and in the presence of Laponite $\mathrm{RD}, \mathrm{Fe}$ powder, graphene oxide, and $\mathrm{C}_{12} \mathrm{E}_{6}$ surfactant. None of these impurities had a significant positive impact on the rate or quantity of hydrate formation (see Figure S5). This suggests that if formation is occurring at the surface of the pressure cylinder, then this is likely due to an enhanced methane concentration, which is also likely the case at the gas/liquid interface.

3.2. Molecular Level Mechanism of Hydrate Formation from Molecular Dynamics Simulations. To 

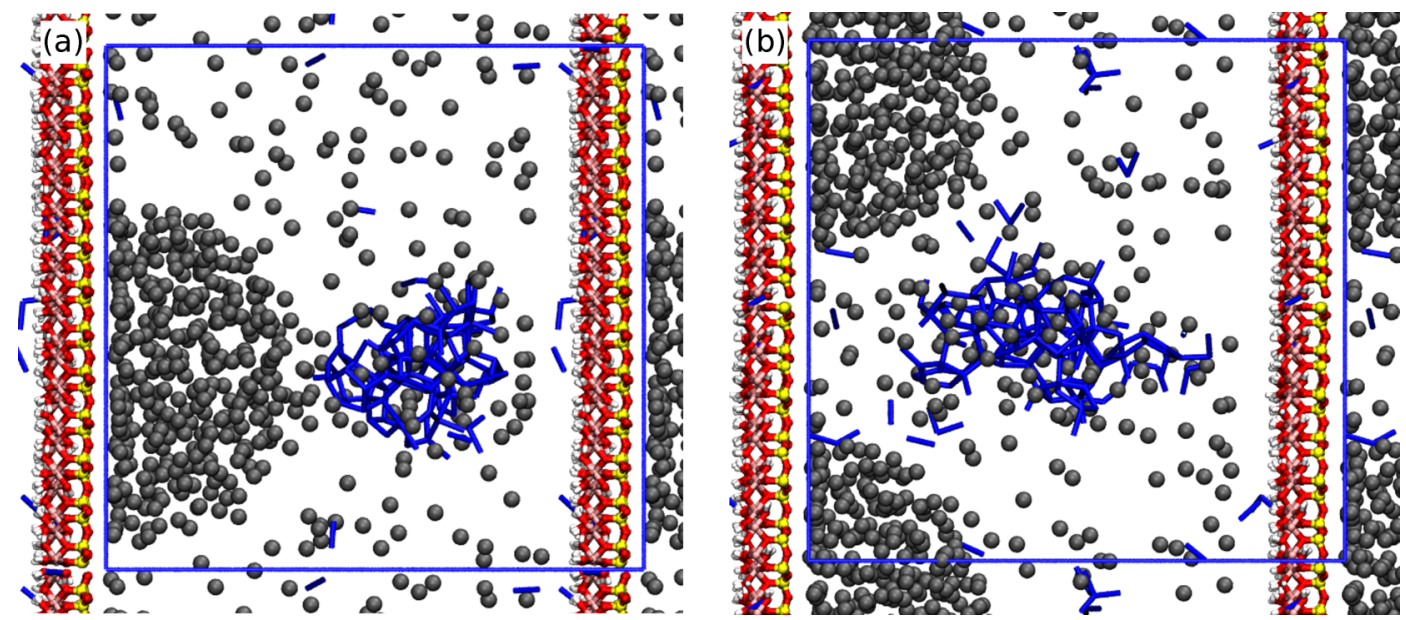

Figure 3. Snapshot of hydrate nucleation in the presence of kaolinite. Methane carbon atoms are shown by gray spheres, "hydrate-like" oxygen atoms are depicted by blue bonds (for clarity, other water molecules are not shown). Oxygen atoms of the kaolinite are shown in red, hydrogen in white, aluminum in pink and silicon in yellow. Nucleation is observed away from the kaolinite surface. The snapshots shown in panels a and $b$ were initiated from different configurations.
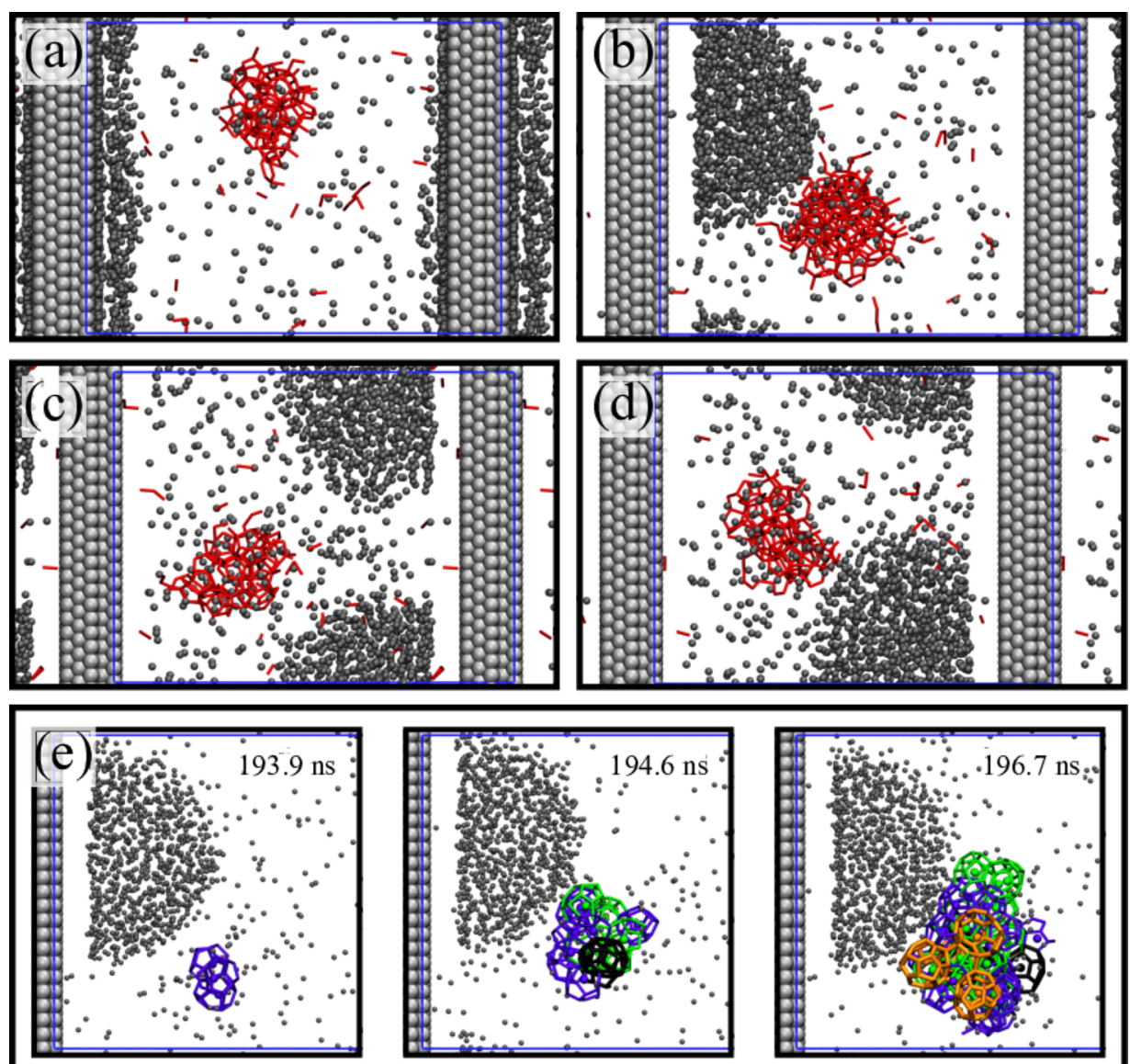

Figure 4. Methane hydrate formation in the presence of solid surfaces occurs away from the surface, as seen in snapshots from MD simulations. Results for a range of water surface interaction strengths are shown, measured by the adsorption energy of a single water molecule to the surface. The adsorption energies are (in kcal/mol): (a) 0.82; (b) 8.01; (c) 15.46; and (d) 20.11. The large light-gray spheres show the surface atoms, the small dark-gray spheres show methane molecules and bonds between "hydrate-like" water molecules are shown by red lines. (For clarity, the remaining water molecules are not shown.) Regardless of the hydrophilicity, nucleation is always observed away from the fcc surface. The blue lines show the boundary of the periodic simulation cell: the area of the surface was approximately $6.1 \times 5.7 \mathrm{~nm}^{2}$ and the distance normal to the surface was allowed to fluctuate to maintain constant pressure (approximately $7 \mathrm{~nm}$ ). In panel e, we show time-resolved snapshots (time indicated in top right corner of each panel) of the nucleation event shown in panel b. Hydrate cages are colored: violet, $5^{12}$; green, $5^{12} 6^{2}$; black, $5^{12} 6^{3}$; and orange $5^{12} 6^{4}$.

provide insight into these experimental results, we have also performed a series of $\mathrm{MD}$ simulations. We begin by discussing results from our simulations of an all-atom representation of a water/methane/kaolinite system. In total, we performed ten 
simulations, starting from configurations in which the methane/ water interface was planar. In eight of these, this fluctuating planar interface was stable on the microsecond time scale, and hydrate nucleation was not observed. In two of the simulations, however, a spontaneous fluctuation resulted in the formation of a cylindrical nanobubble, which led to an increase in dissolved methane concentration due to Laplace pressure effects. ${ }^{13}$ In Figure 3, we present snapshots from the two successful nucleation events. By using the CHILL+ algorithm, an algorithm based on local bond order parameters, to identify "clathrate-like" water molecules, ${ }^{55}$ we can clearly see that nucleation is occurring away from either kaolinite surface in both instances. (Hydroxyl oxygen atoms belonging to the kaolinite were also included in the CHILL+ analysis.) The results from the simulations with the kaolinite surface are consistent with our experimental finding that the addition of mineral particles has little effect on the formation of methane hydrate. However, the cost of a model that attempts to capture the atomistic details of a surface similar to those probed experimentally is computationally demanding, making it difficult to go beyond the qualitative insight obtained from the snapshots presented in Figure 3.

To go further, we have also investigated methane hydrate formation with a coarse grained description of water and methane in the presence of two types of model surface: the (111) surface of a fcc crystal; and a graphene sheet. While with these types of models we are not directly attempting to describe nucleation on any particular surface, by altering the interaction strength of the surface with the water molecules, and by using two distinctly different types of surface, we are able to investigate both the effect of surface hydrophilicity and surface structure on hydrate nucleation. This allows us to gain general insight into the role of surface hydrophilicity in the nucleation of methane hydrate, as a complement to the neutron scattering experiments and the atomistic simulations that probe the formation of methane hydrate in the presence of specific surfaces.

Whereas the fcc and graphene surfaces facilitate ice formation by distinctly different mechanisms, ${ }^{42-46,50,51}$ in the case of methane hydrate, however, no qualitative differences between the two surfaces is observed. We therefore limit ourselves here to discussion of results from the fcc surface, and provide the results from the graphene surface in the S.I. (Figures S8 and S9). In Figure 4, we show representative snapshots of nucleation events in the presence of the fcc surface with four different hydrophilicities (results from a further two hydrophilicities are presented in Figures S6 and S7). In each snapshot, we are showing bonds between water molecules that are classed as "hydrate-like" according to the CHILL+ algorithm. ${ }^{55}$ Although our liquid film is relatively thin (ca. 7 $\mathrm{nm}$ ), it can be clearly seen that in all cases, the mechanism of hydrate formation is the same, and that nucleation occurs away from the solid surface. Moreover, the nucleation mechanism appears to follow that reported in previous simulation studies of homogeneous nucleation. ${ }^{9,10}$ This is exemplified by Figure 4e, which shows time-resolved snaphots of one of the nucleation events. Here we can see the initial formation of face-sharing $5^{12}$ cages, followed by the subsequent formation of larger cages. Overall, from both the all-atom and coarse grained simulations, we see that the presence of solid surfaces has little effect on the molecular mechanism by which methane hydrate forms.

3.3. Discussion. The fact that in both our experiments and simulations the introduction of impurity particles has little effect on methane hydrate formation is, at face value, surprising. The explanation we provide, however, is straightforward and is due to the different chemical natures of methane and water. Previous simulation studies ${ }^{9,10,13}$ have shown that for hydrate nucleation to proceed, the dissolved methane molecules need to aggregate as solvent-separated pairs, requiring the water and the methane to be mixed. Whereas water molecules are polar and are able to form relatively strong hydrogen bonds, methane molecules are nonpolar and interact primarily through much weaker dispersion interactions. The different chemical natures of methane and water therefore make it unlikely that the surface of a dissolved particle will simultaneously display a strong affinity for both species so as to promote their mixing at the microscopic level. Such behavior is displayed in Figure 5, which
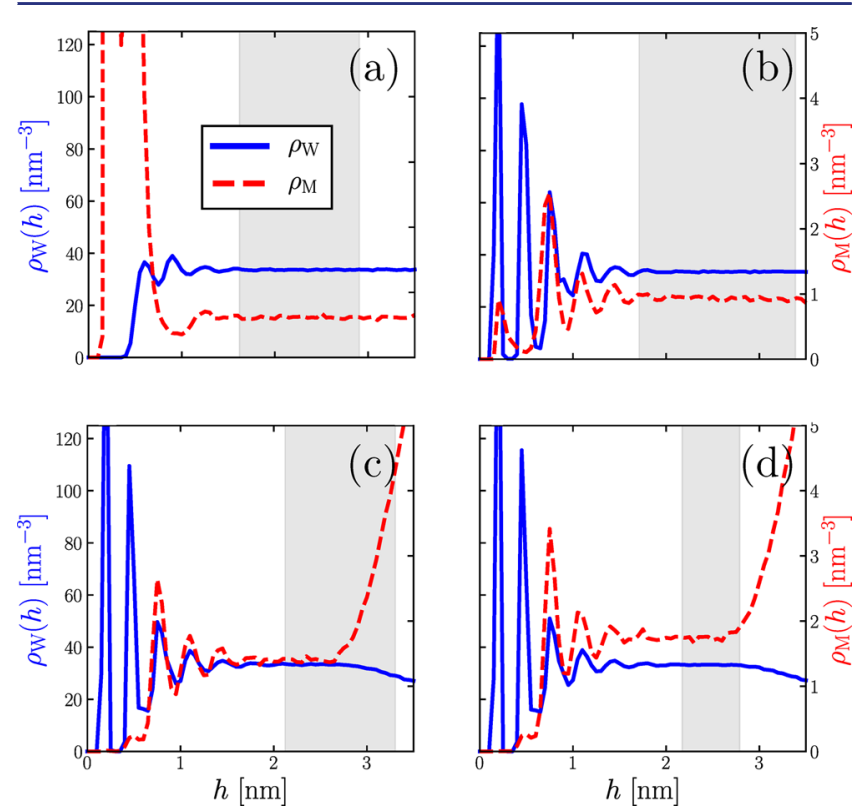

Figure 5. Surfaces either prefer water or methane but not both, as illustrated by the densities of water $\rho_{\mathrm{W}}(h)$ (blue) and methane $\rho_{\mathrm{M}}(h)$ (red) vs distance $h$ from the fcc surfaces shown in Figure 4 . The adsorption energy of a single water molecule to the surface is (in kcal/ mol): (a) 0.82; (b) 8.01; (c) 15.46; and (d) 20.11. At the most hydrophobic surface (a), methane forms a contact layer with little water found close to the surface. At the more hydrophilic surfaces (bd), the situation is reversed. The shaded gray areas span the range of $h$ at which nucleation was observed: in all cases, $h>1 \mathrm{~nm}$, i.e., nucleation directly at the solid surface is not observed.

shows the density at $250 \mathrm{~K}$ and 900 atm of both water and methane above the fcc surfaces shown in Figure 4. At the most hydrophobic surface, Figure 5a, the methane is in contact with the surface, with negligible water content within $0.5 \mathrm{~nm}$. As the surface hydrophilicity increases, Figure $5 b-d$, we find that the situation is reversed, with methane generally excluded from the surface. (The small peak in the methane density near the surface shown in Figure $5 \mathrm{~b}$ corresponds to an average of fewer than five methane molecules within our approximately $6.1 \times 5.7$ $\mathrm{nm}^{2}$ surface simulation cell.) The shaded gray areas in Figure 5 span the range of approximate heights above the surface at which nucleation was observed (details given in the S.I.), and clearly show that nucleation is always observed at distances $h>$ $1 \mathrm{~nm}$ from the surface. Despite the fact that nucleation does not occur at the surface, we show in Table S2 that some surfacedependence is observed for the temperature at which 
nucleation occurs. Though this might be interpreted as evidence of the surfaces promoting nucleation, inspection of Figure 5 (see also Figures S7 and S9, and Table S2) shows that this dependence is correlated with an increase in the concentration of dissolved methane. Due to finite size effects on the water-methane interfacial curvature, ${ }^{13}$ it is possible such dependence disappears in the thermodynamic limit. It does suggest a possibility, however, that although the surfaces do not faciliate methane hydrate formation directly, they may do so indirectly, if they have a significant effect on the uptake of methane into solution. This is consistent with recent experimental results, which show that confinement effects within cavities of activated carbon can also alter local methane concentration and promote hydrate formation. ${ }^{32}$

As discussed in the section 1, a motivation for this work was the finding from a previous simulation study that the homogeneous nucleation rate of methane gas hydrate under realistic conditions was effectively zero. ${ }^{33}$ Cha et al. ${ }^{22}$ had previously reported that bentonite significantly enhanced hydrate formation (although for a gas mixture rather than pure methane), as well as raising the dissociation temperatures and pressures compared to the pure water and gas system. On the other hand, Uchida et al. ${ }^{56}$ found that for low water content, bentonite substantially decreased the dissociation temperature of the hydrate, and for higher water contents, either had no effect or slightly increased the dissociation temperature of the hydrate by at most $0.5 \mathrm{~K}$. The results presented in this study suggest that mineral impurity particles are not the most likely source of heterogeneous nucleation sites. Although our simulations use a high driving force, previous experience with ice nucleation ${ }^{42-46}$ suggests that we would still expect to see an effect of the surfaces if they were to promote nucleation. Our neutron scattering experiments also indicate that particulate matter has little effect on methane hydrate formation, and our test experiments suggest that if it is facilitated by the container wall, then this is likely due to an enhanced concentration of methane at the interface. This appears to be supported by X-ray tomographic microscopy images $^{57}$ of the early stages of methane hydrate formation in the ocean, which showed methane hydrate formation at the surfaces of gas bubbles, where dissolved gas concentrations are likely to be high. Similarly, Chaouachi et al. ${ }^{58}$ used X-ray tomographic microscopy to image xenon gas hydrate formation in quartz and glass bead matrices. In samples with no history of gas hydrate formation, the crystals were seen to form at the gas/liquid interface. On the other hand, in samples prepared by melting gas hydrate, such that the resulting fluid was gas enriched, formation was observed in the bulk. Interestingly, a liquid film of several micrometers was observed between the solid surfaces and the resulting crystal. It was also observed that for hydrophobic quartz, a layer of gas instead separated the hydrate from the surface, suggesting that the hydrate crystal, which is generally considered "hydrophilic", prefers to form a low energy interface with the gas instead of quartz.

\section{CONCLUSIONS}

Using both neutron scattering experiments and MD simulations, we have found that methane hydrate formation is insensitive to the addition of impurity particles. In all of our MD simulations, both atomistic and coarse grained, we always observe nucleation occurring away from the surface. We attribute this observation to the different chemical natures of methane and water making it unlikely that the surface of a dissolved particle will simultaneously display a strong affinity for both species so as to promote their mixing at the microscopic level. Although experimentally we cannot preclude the formation of methane hydrate on the surface of the pressure cylinder, our test experiments suggest that if this is the case, then this is likely due to an enhanced methane concentration, rather than the surface chemistry of the cylinder intrinsically promoting methane hydrate formation. It is also likely that methane hydrate is forming close to the gas/liquid interface, where dissolved methane concentrations are likely to be higher.

In this study, we have investigated the effect of impurity particles only on the formation of methane hydrate. In the case that other gas molecules are present, one may not necessarily expect to observe the same behavior as we have seen here, even though the nucleation mechanisms for larger or more miscible guest molecules share many similarities with that of small guest molecules like methane. ${ }^{59}$ For more hydrophilic or watersoluble guest molecules (e.g., THF or $\mathrm{CO}_{2}$ ), impurity particles may indeed promote hydrate formation in a similar fashion to heterogeneous ice nucleation. ${ }^{28-31,60}$ We finish with a comment regarding hydrate inhibition. One mechanism by which kinetic inhibitors function is to bind to surfaces of the hydrate crystal. ${ }^{4}$ If methane hydrate formation is occurring away from the mineral surfaces, then one possible way to improve the performance of such inhibitors is to weaken their affinity for adsorption to the surfaces of dissolved solid particles.

\section{ASSOCIATED CONTENT}

\section{S Supporting Information}

The Supporting Information is available free of charge on the ACS Publications website at DOI: $10.1021 /$ jacs.7b12050.

Further details of experimental and simulation protocols; additional information of experimental samples; details of calculating the height above surface at which nucleation is observed in simulations; further simulation results (PDF)

\section{AUTHOR INFORMATION}

\section{Corresponding Authors}

*n.skipper@ucl.ac.uk

*angelos.michaelides@ucl.ac.uk

ORCID

Stephen J. Cox: 0000-0003-2708-8711

Alan K. Soper: 0000-0002-7903-8356

Angelos Michaelides: 0000-0002-9169-169X

\section{Notes}

The authors declare no competing financial interest.

\section{ACKNOWLEDGMENTS}

This work was supported in part by the European Research Council under the European Union's Seventh Framework Programme (FP/2007-2013)/ERC Grant Agreement number 616121 (HeteroIce project). A.M. is also supported by the Royal Society through a Royal Society Wolfson Research Merit Award. We are grateful for computational resources provided by the London Centre for Nanotechnology and the Materials Chemistry Consortium through the EPSRC grant number EP/ L000202. The authors also acknowledge that the work presented here made use of the IRIDIS High Performance Computing facility provided via the Centre for Innovation (CfI). The CfI is formed from the universities of Bristol, 
Oxford, Southampton and University College London. We thank Chris Goodway, Mark Kibble and Paul McKintyre from the ISIS Facility sample environment group. Experiments were conducted under ISIS awards RB1320495, RB1410533, RB1400022, and the ISIS Collaborative R\&D (ICRD) Scheme, award ICRD031.

\section{REFERENCES}

(1) Sloan, E. D.; Koh, C. A. Clathrate Hydrates of Natural Gases, Third ed.; CRC Press: Boca Raton, Florida, USA, 2008.

(2) Klauda, J. B.; Sandler, S. I. Energy Fuels 2005, 19, 459-470.

(3) Sloan, E. D. Nature 2003, 426, 353-359.

(4) Yagasaki, T.; Matsumoto, M.; Tanaka, H. J. Am. Chem. Soc. 2015, 137, 12079-12085.

(5) Warrier, P.; Khan, M. N.; Srivastava, V.; Maupin, C. M.; Koh, C. A. J. Chem. Phys. 2016, 145, 211705.

(6) Buchanan, P.; Soper, A. K.; Thompson, H.; Westacott, R. E.; Creek, J. L.; Hobson, G.; Koh, C. A. J. Chem. Phys. 2005, 123, 164507.

(7) Koh, C. A.; Wisbey, R. P.; Wu, X.; Westacott, R. E.; Soper, A. K. J. Chem. Phys. 2000, 113, 6390-6397.

(8) Koh, C. A.; Savidge, J. L.; Tang, C. C. J. Phys. Chem. 1996, 100, 6412-6414.

(9) Walsh, M. R.; Koh, C. A.; Sloan, E. D.; Sum, A. K.; Wu, D. T. Science 2009, 326, 1095-1098.

(10) Jacobson, L. C.; Hujo, W.; Molinero, V. J. Am. Chem. Soc. 2010, 132, 11806-11811.

(11) Barnes, B. C.; Knott, B. C.; Beckham, G. T.; Wu, D. T.; Sum, A. K. J. Phys. Chem. B 2014, 118, 13236-13243.

(12) Lauricella, M.; Meloni, S.; English, N. J.; Peters, B.; Ciccotti, G. J. Phys. Chem. C 2014, 118, 22847-22857.

(13) Walsh, M. R.; Beckham, G. T.; Koh, C. A.; Sloan, E. D.; Wu, D. T.; Sum, A. K. J. Phys. Chem. C 2011, 115, 21241-21248.

(14) Bi, Y.; Li, T. J. Phys. Chem. B 2014, 118, 13324-13332.

(15) Jiménez-Ángeles, F.; Firoozabadi, A. J. Phys. Chem. C 2015, 119, 8798-8804.

(16) Vatamanu, J.; Kusalik, P. G. J. Am. Chem. Soc. 2006, 128, 15588-15589.

(17) Sarupria, S.; Debenedetti, P. G. J. Phys. Chem. Lett. 2012, 3, 2942-2947.

(18) Hawtin, R. W.; Quigley, D.; Rodger, P. M. Phys. Chem. Chem. Phys. 2008, 10, 4853-4864.

(19) Jacobson, L. C.; Molinero, V. J. Am. Chem. Soc. 2011, 133, 6458-6463.

(20) Liang, S.; Kusalik, P. G. J. Phys. Chem. B 2013, 117, 1403-1410. (21) Guggenheim, S.; Koster van Groos, A. F. Geology 2003, 31, 653-656.

(22) Cha, S. B.; Ouar, H.; Wildeman, T. R; Sloan, E. D. J. Phys. Chem. 1988, 92, 6492-6494.

(23) Sun, R.; Duan, Z. Chem. Geol. 2007, 244, 248-262.

(24) Zhang, Y.; Debenedetti, P. G.; Prud'homm, R. K.; Pethica, B. A. J. Phys. Chem. B 2004, 108, 16717-16722.

(25) Nguyen, A. H.; Koc, M. A.; Shepherd, T. D.; Molinero, V. J. Phys. Chem. C 2015, 119, 4104-4117.

(26) Pirzadeh, P.; Kusalik, P. G. J. Am. Chem. Soc. 2013, 135, 72787287.

(27) Poon, G. G.; Peters, B. Cryst. Growth Des. 2013, 13, 4642-4647.

(28) Bai, D.; Chen, G.; Zhang, X.; Wang, W. Langmuir 2011, 27, 5961-5967.

(29) Bai, D.; Chen, G.; Zhang, X.; Wang, W. Langmuir 2012, 28, $7730-7736$

(30) Bai, D.; Chen, G.; Zhang, X.; Sum, A. K.; Wang, W. Sci. Rep. 2015, 5, 12747.

(31) Kyung, D.; Lim, H.-K.; Kim, H.; Lee, W. Environ. Sci. Technol. 2015, 49, 1197-1205.

(32) Casco, M. E.; Silvestre-Albero, J.; Ramírez-Cuesta, A. J.; Rey, F.; Jordá, J. L.; Bansode, A.; Urakawa, A.; Peral, I.; Martínez-Escandell, M.; Kaneko, K.; Rodríguez-Reinoso, F. Nat. Commun. 2015, 6, 6432.
(33) Knott, B. C.; Molinero, V.; Doherty, M. F.; Peters, B. J. Am. Chem. Soc. 2012, 134, 19544-19547.

(34) Casco, M. E.; Rey, F.; Jordá, J. L.; Rudić, S.; Fauth, F.; MartínezEscandell, M.; Rodríguez-Reinoso, F.; Ramos-Fernández, E. V.; Silvestre-Albero, J. Chem. Sci. 2016, 7, 3658-3666.

(35) Moudrakovski, I. L.; McLaurin, G. E.; Ratcliffe, C. I.; Ripmeester, J. A. J. Phys. Chem. B 2004, 108, 17591-17595.

(36) Muller-Bongartz, B.; Wildeman, T. R.; Sloan, E. D., Jr. A Hypothesis For Hydrate Nucleation Phenomena. The Second International Offshore and Polar Engineering Conference, San Francisco, CA, June 14-19, 1992; International Society of Offshore and Polar Engineers, 1992; ISOPE-I-92-089.

(37) Sloan, E. D.; Fleyfel, F. AIChE J. 1991, 37, 1281-1292.

(38) Radhakrishnan, R.; Trout, B. L. J. Chem. Phys. 2002, 117, 17861796.

(39) Dec, S. F.; Bowler, K. E.; Stadterman, L. L.; Koh, C. A.; Sloan, E. D. J. Am. Chem. Soc. 2006, 128, 414-415.

(40) Bowron, D. T.; Soper, A. K.; Jones, K.; Ansell, S.; Birch, S.; Norris, J.; Perrott, L.; Riedel, D.; Rhodes, N. J.; Wakefield, S. R.; Botti, A.; Ricci, M.-A.; Grazzi, F.; Zoppi, M. Rev. Sci. Instrum. 2010, 81, 033905 .

(41) Benmore, C. J.; Soper, A. K. The SANDALS Manual: a Guide to Performing Experiments on the Small Angle Neutron Diffractometer for Amorphous and Liquid Samples at ISIS, Version 1.0; RAL-TR-1998006; RAL Technical Reports: Didcot U.K., 1998; p 1.

(42) Cox, S. J.; Kathmann, S. M.; Slater, B.; Michaelides, A. J. Chem. Phys. 2015, 142, 184704.

(43) Cox, S. J.; Kathmann, S. M.; Slater, B.; Michaelides, A. J. Chem. Phys. 2015, 142, 184705.

(44) Fitzner, M.; Sosso, G. C.; Cox, S. J.; Michaelides, A. J. Am. Chem. Soc. 2015, 137, 13658-13669.

(45) Lupi, L.; Hudait, A.; Molinero, V. J. Am. Chem. Soc. 2014, 136, 3156-3164.

(46) Lupi, L.; Molinero, V. J. Phys. Chem. A 2014, 118, 7330-7337.

(47) Molinero, V.; Moore, E. B. J. Phys. Chem. B 2009, 113, 40084016.

(48) Jacobson, L. C.; Molinero, V. J. Phys. Chem. B 2010, 114, 73027311.

(49) Plimpton, S. J. Comput. Phys. 1995, 117, 1-19.

(50) Cabriolu, R.; Li, T. Phys. Rev. E 2015, 91, 052402.

(51) Bi, Y.; Cabriolu, R; Li, T. J. Phys. Chem. C 2016, 120, 15071514.

(52) Hess, B.; Kutzner, C.; van der Spoel, D.; Lindahl, E. J. Chem. Theory Comput. 2008, 4, 435.

(53) Pruppacher, H. R.; Klett, J. D. Microphysics of Clouds and Precipitation - Second Revised and Enlarged ed. with an Introduction to Cloud Chemistry and Cloud Electricity; Kluwer Academic Publishers: Dordrecht, The Netherlands, 1997.

(54) Murray, B. J.; O’Sullivan, D.; Atkinson, J. D.; Webb, M. E. Chem. Soc. Rev. 2012, 41, 6519-6554.

(55) Nguyen, A. H.; Molinero, V. J. Phys. Chem. B 2015, 119, 93699376.

(56) Uchida, T.; Takeya, S.; Chuvilin, E. M.; Ohmura, R.; Nagao, J.; Yakushev, V. S.; Istomin, V. A.; Minagawa, H.; Ebinuma, T.; Narita, H. J. Geophys. Res.: Solid Earth 2004, 109, B05206.

(57) Klapp, S. A.; Enzmann, F.; Walz, P.; Huthwelker, T.; Tuckermann, J.; Schwarz, J.-O.; Pape, T.; Peltzer, E. T.; Mokso, R.; Wangner, D.; Marone, F.; Kersten, M.; Bohrmann, G.; Kuhs, W. F.; Stampanoni, M.; Brewer, P. G. Geo-Mar. Lett. 2012, 32, 555-562.

(58) Chaouachi, M.; Falenty, A.; Sell, K.; Enzmann, F.; Kersten, M.; Haberthur, D.; Kuhs, W. F. Geochem., Geophys., Geosyst. 2015, 16, $1711-1722$.

(59) Jacobson, L. C.; Hujo, W.; Molinero, V. J. Phys. Chem. B 2010, 114, 13796-13807.

(60) Zeng, H.; Wilson, L. D.; Walker, V. K.; Ripmeester, J. A. J. Am. Chem. Soc. 2006, 128, 2844-2850. 